\title{
Fiber Modeling and Clustering Based on Neuroanatomical Features
}

\author{
Qian Wang ${ }^{1,2}$, Pew-Thian Yap ${ }^{2}$, Guorong $\mathrm{Wu}^{2}$, and Dinggang Shen ${ }^{2}$ \\ ${ }^{1}$ Department of Computer Science, University of North Carolina at Chapel Hill \\ qianwang@cs. unc. edu \\ ${ }^{2}$ Department of Radiology and BRIC, University of North Carolina at Chapel Hill \\ \{ptyap, grwu, dgshen\} @med.unc. edu
}

\begin{abstract}
DTI tractography allows unprecedented understanding of brain neural connectivity in-vivo by capturing water diffusion patterns in brain white-matter microstructures. However, tractography algorithms often output hundreds of thousands of fibers, rendering the computation needed for subsequent data analysis intractable. A remedy is to group the fibers into bundles using fiber clustering techniques. Most existing fiber clustering methods, however, rely on fiber geometrical information only by viewing fibers as curves in the $3 \mathrm{D}$ Euclidean space. The important neuroanatomical aspect of the fibers is mostly ignored. In this paper, neuroanatomical information is encapsulated in a feature vector called the associativity vector, which functions as the "fingerprint" for each fiber and depicts the connectivity of the fiber with respect to individual anatomies. Using the associativity vectors of fibers, we model the fibers as observations sampled from multivariate Gaussian mixtures in the feature space. An expectation-maximization clustering approach is then employed to group the fibers into 16 major bundles. Experimental results indicate that the proposed method groups the fibers into anatomically meaningful bundles, which are highly consistent across subjects.
\end{abstract}

\section{Introduction}

Diffusion Tensor Imaging (DTI) has become a popular imaging modality in exploring brain circuitry in-vivo by capturing water diffusion patterns in brain tissues. Water molecules are more likely to diffuse parallel along neural pathways, since myelin sheaths of axons act as barriers and restrict the mobility of water molecules along directions perpendicular to the neural pathways. In a DT image, each voxel records the anisotropic water diffusion pattern at a specific location of the brain using a second-order tensor. The principal eigenvector of the tensor, corresponding to the maximal eigenvalue, indicates the major direction along which water molecules are diffusing. By tracing along these directions, neural tracts can thus be delineated via a process called tractography [1].

Several fiber tractography algorithms have been proposed in the literature. An intuitive and straightforward streamline-based tractography approach begins fiber tracing from a given seed point and grows smoothly along the principal eigenvectors of the underlying tensor field. This tracking procedure continues until either the fiber enters regions with low fractional anisotropy (FA), where directional information is 
no longer reliable, or when the curvature of the fiber pathway is too large. With whole brain seeding, a typical fiber tractography algorithm can yield fibers numbering in the order of $10^{3}-10^{6}$.

Fiber tractography provides an effective approach in visualizing and analyzing brain connectivity, and thus is important in clinical applications. However, the task of analyzing fibers yielded by tractography is non-trivial. The massive amount of fibers often renders subsequent analyses difficult, and makes information provided by the fibers not immediately decipherable. One possible solution is to automatically partition fibers into dozens of bundles, each of which contains fibers that are characterized by similar structural and functional behaviors. Analyses can then be performed on the bundles, instead of the individual fibers.

Existing fiber clustering methods are mostly geometry based approaches. Fibers are typically viewed as a set of curves in the 3D space. In [2], for example, point-topoint correspondences are first established between fibers. The similarity between a pair of fibers is then defined as the ratio of the length of the corresponding segment over the entire fiber length. The correspondence ratio between fibers is maximized when the two fibers are identical, and vanishes if pairwise correspondence is minimal. Similar ideas based on point-to-point correspondences between fibers are applied in $[3,4]$. Fiber similarity can also be calculated by counting the number of voxels that the two fibers share [5]. Other approaches involve extracting features from the spatial distributions or parametric representations of fibers [6-9]. The Hausdorff distance, as well as its variants, has also been widely applied to fiber clustering [10-14].

Geometry based methods, however, ignores the neuroanatomical characteristics of fibers and results in bundles which are not clear for interpretations from an anatomical and connectivity point of view. It is therefore important to incorporate anatomical information when performing fiber clustering. For this purpose, Maddah et. al. [7] report an atlas where individual bundles have already been delineated and labeled by experts. Based on this atlas, fibers are clustered and labeled with the known bundles according to geometric similarities. Alternative approaches can also be found in $[15$, 16], where bundles are tagged according to the regions they connect.

In this paper, we propose a novel fiber clustering scheme that is solely based on the neuroanatomical features of fibers. After performing whole brain tractography, we parcellate the brain with a number of ROIs and diffuse the ROIs according to the underlying fibers. ROI diffusion helps more robustly determine the relationship between the anatomical regions (as delineated by the ROIs) and the fibers by using this fuzzy formulation. We are then able to acquire the associativity vector for each fiber to describe its connectivity pattern with respect to all ROIs of different anatomies. Each entry of the associativity vector represents the likelihood of the fiber being connected to a particular ROI. We further model each bundle as a multivariate Gaussian mixture based on the associativity vectors of the fibers belonging to the bundle. An expectation-maximization (EM) approach is thus employed to group the fibers as belonging to one of the bundles or as outliers. Experimental results show that the proposed method can achieve consistent clustering results across individual subjects, implying its potential usage in analyzing a population of DTI data. 


\section{Method}

We will first define the associativity vector (Section 2.1) and then introduce the multivariate-Gaussian-mixture-based EM clustering approach (Section 2.2).

\subsection{Neuroanatomical Features of Fibers}

Fibers connect different anatomical regions of the brain, forming a connectivity pattern that describes brain circuitry. For annotating different regions of the brain, we warp the "Eve" atlas [17] (called atlas for brevity in the rest of the paper) to the native space of each individual subject via non-rigid registration. The atlas contains T1 image, tensor field, and a set of manually delineated ROIs for important anatomical structures of the brain.

Suppose that there are M ROIs, we then define the associativity vector $L_{i}=$ $\left(\ell_{i 1}, \ell_{i 2}, \cdots, \ell_{i \mathrm{M}}\right)$ for fiber $i$, where the entry $\ell_{i j}$ measures the relationship between fiber $i$ and the $j$-th ROI. Entry $\ell_{i j}$ can be set to 1 if any segment of fiber $i$ lies within ROI $j$, and left unset otherwise. However, this binary formulation, though simple, would result in sparse associativity vectors, pose challenges in estimating fiber distances, and increase the tendency of the clustering algorithms to be trapped in local minima. Moreover, fibers that are prematurely terminated a little short of reaching the ROI due to imaging noise might also be penalized.

In view of this, we propose a fuzzy ROI spatial confidence map by allowing the ROI to grow, or to diffuse, along directions indicated by the fibers. For this purpose, we adopt the fast marching (FM) approach [18]. As illustrated in Fig. 1(a), the initial ROI (dark red area) starts diffusing since time $t_{s}$ and terminates at $t_{e}$. The initial ROI surface (dashed green curve) moves outward following the underlying fibers from upper-left to lower-right. At location $\mathrm{x}$ on the surface, diffusion proceeds along the surface normal direction. The contribution of a fiber to the ROI diffusion at $\mathrm{x}$ is defined as the inner product of the tangential direction of the fiber and the surface normal. Summing up contributions from all fibers traversing $\mathrm{x}$, we obtain the overall diffusion velocity $v(\mathrm{x})$. By iteratively solving the Eikonal equation $v\left\|\nabla_{\mathrm{x}} \tau\right\|=1$ in FM, we then acquire the time value when a certain location is traversed by the ROI surface. The location geodesically closer to the initial ROI will be traversed by the surface at an earlier time. We thus invert all recorded time values and rescale them to the range of $[0,1]$, giving a spatial confidence map of the ROI. In Fig. 1(a), for example, dark red indicates locations near the initial ROI and dark blue indicates locations far away.

We have also provided a real example in Fig. 1(b)-(d). In Fig. 1(b), the bundle connecting the left superior-frontal gyrus (SPG-L) to the right superior-frontal gyrus (SPG-R) is overlaid in red on the FA map. The ROI of SPG-L diffuses accordingly, and results in the spatial confidence map in panel (c) where the dark red area is the initial ROI. The diffusion pattern of SPG-R is similar and displayed in Fig. 1(d).

All ROIs are diffused according to the fibers given by whole-brain tractography, and resulting in their own spatial confidence maps. We allow the same amount of time for diffusion of all ROIs, in order to assure that the re-scaled spatial confidence maps are comparable. For the associativity vector $L_{i}$ of fiber $i$, the entry $\ell_{i j}$ is 
defined as the maximal spatial confidence value that fiber $i$ comes upon the spatial confidence map of ROI $j$. The atlas consists of 130 ROIs (usually at the scale of gyrus/sulcus), but we only need to detect 16 major fiber bundles as in the following. To reduce the redundancy between entries of the associativity vector, we integrate smaller ROIs into larger ones. In particular, the ROIs we have used in this work include the left/right frontal lobes, the left/right central areas, the left/right parietal lobes, the left/right occipital lobes, the left/right temporal lobes, the left/right subcortical areas, the brainstem, and the cerebellum.

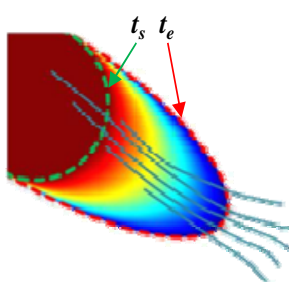

(a)

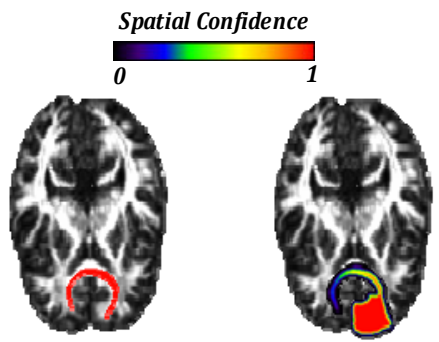

(b) (c)

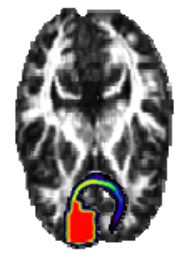

(d)

Fig. 1. In (a), the ROI is diffused along the underlying bundle. The diffusion starts from the green dashed curve $\left(\right.$ at $t_{s}$ ) and terminates at the red dashed curve (at $t_{e}$ ). The spatial confidence map of the ROI can thus be calculated using the traversing time of the diffusion surface. In (b), the bundle connecting SPG-L and SPG-R is overlaid in red on the FA map. The spatial confidence maps of SPG-L and SPG-R after diffusion according to the bundle in (b), are shown in (c) and (d), respectively.

\subsection{Bundle Modeling and Clustering}

Fibers in the same bundle typically share similar neural pathways and generate highly correlated associativity vectors. As the result, we can model the distribution of fibers in a bundle using their associativity vectors with a multivariate Gaussian mixture. An expectation-maximization (EM) approach based on parametric bundle models can then be used for fiber clustering. For initialization of the EM based clustering, we have manually reproduced 16 major bundles (listed in Table 1) in the atlas space following [19]. Parameters estimated from these bundles will work as constraints to guide fiber clustering in each subject. The fibers of the subject will be either grouped into one of the 16 bundles or tagged as outliers. The parameters for the multivariate Gaussian mixture representing each bundle $k(1 \leq k \leq K)$ include the mean associativity vector $\mu_{k}$ and the covariance matrix $S_{k}$. For fiber $i(1 \leq i \leq N)$, we denote its associativity vector as $L_{i}$ and its membership to bundle $k$ as $\omega_{i k}$.

\section{E-step:}

According to Bayes rule, the membership of fiber $i$ to bundle $k$ can be estimated as:

$$
\omega_{i k} \leftarrow \frac{\alpha_{k} \cdot N_{k}\left(L_{i} \mid \mu_{k}, S_{k}\right)}{\sum_{m=1}^{K} \alpha_{m} \cdot N_{m}\left(L_{i} \mid \mu_{m}, S_{m}\right)}
$$


where $N_{k}\left(L_{i} \mid \mu_{k}, S_{k}\right)$ is the multivariate Gaussian distribution centered at $\mu_{k}$ with covariance $S_{k}$. For the first E-step, the parameters $\left(\mu_{k}, S_{k}\right)$ are directly introduced from the manually delineated bundles in the atlas, while $\alpha_{k}$ denotes the number of fibers in bundle $k$.

Table 1. The list of the 16 target bundles

\begin{tabular}{ll|ll}
\hline Abbr. & Description & Abbr. & Description \\
\hline Fminor & Forceps minor & SLF-R & Superior longitudinal fasciculus (right) \\
Fmajor & Forceps major & SLF-L & Superior longitudinal fasciculus (left) \\
CST-R & Corticospinal tract (right) & ILF-R & Inferior longitudinal fasciculus (right) \\
CST-L & Corticospinal tract (left) & ILF-L & Inferior longitudinal fasciculus (left) \\
ATR-R & Anterior thalamic radiation (right) & UNC-R & Uncinate fasciculus (right) \\
ATR-L & Anterior thalamic radiation (left) & UNC-L & Uncinate fasciculus (left) \\
IFO-R & Inferior fronto-occipital fasciculus (right) & CB-R & Cingulum (right) \\
IFO-L & Inferior fronto-occipital fasciculus (left) & CB-L & Cingulum (left) \\
\hline
\end{tabular}

\section{M-step:}

The parameters $\left(\mu_{k}, S_{k}\right)$ are updated in the M-step. Based on the membership values estimated in the E-step, we first compute the mixture weighting factor for each bundle $\alpha_{k} \leftarrow \sum_{i=1}^{N} \omega_{i k} / N$ and then the bundle parameters:

$$
\begin{gathered}
\mu_{k} \leftarrow \lambda \mu_{k}+\left(\frac{1-\lambda}{\sum_{i=1}^{N} \omega_{i k}}\right) \sum_{i=1}^{N} \omega_{i k} L_{i} \\
S_{k} \leftarrow\left(\frac{1}{\sum_{i=1}^{N} \omega_{i k}}\right) \sum_{i=1}^{N} \omega_{i k}\left(L_{i}-\mu_{k}\right)\left(L_{i}-\mu_{k}\right)^{t}
\end{gathered}
$$

We set $\lambda$ of (2) to 0.95 to prevent drastic shifts of $\mu_{k}$, which might occur due to large fiber variation. Subsequent E-steps and M-steps will be iteratively executed until convergence. Fibers whose highest memberships are lower than a predefined threshold are regarded as outliers.

\section{Experimental Results}

A total of 15 healthy subjects were used to evaluate the proposed fiber clustering method. The diffusion weighted data were acquired using a Siemens Allegra scanner $\left(\mathrm{b}=1000 \mathrm{~s} / \mathrm{mm}^{2}\right.$, flip angle $90^{\circ}, \mathrm{TR} / \mathrm{TE}=13,640 / 82 \mathrm{~ms}$, matrix $128 \times 128$, FoV $256 \times 256 \mathrm{~mm}^{2}$, slice thickness $2 \mathrm{~mm}, 80$ contiguous slices). In Fig. 2(a), the manually delineated bundles in the atlas space, following protocols in [19], are annotated in different colors. It is worth noting that all right hemisphere bundles are excluded from Fig. 2(a) and only 9 target bundles are shown for visualization. In panels (b)-(e), we show the clustering results of 4 randomly selected subjects. In each panel, callosal fibers (Fminor and Fmajor) are only shown in the top image and excluded in the bottom image for better visualization of other bundles (i.e., CB). In (b)-(e), bundles in the right hemispheres are also removed for visualization clarity, as we have done in (a). Overall, for all 4 subjects shown in Fig. 2, the clustering results are consistent with the atlas and across subjects. 


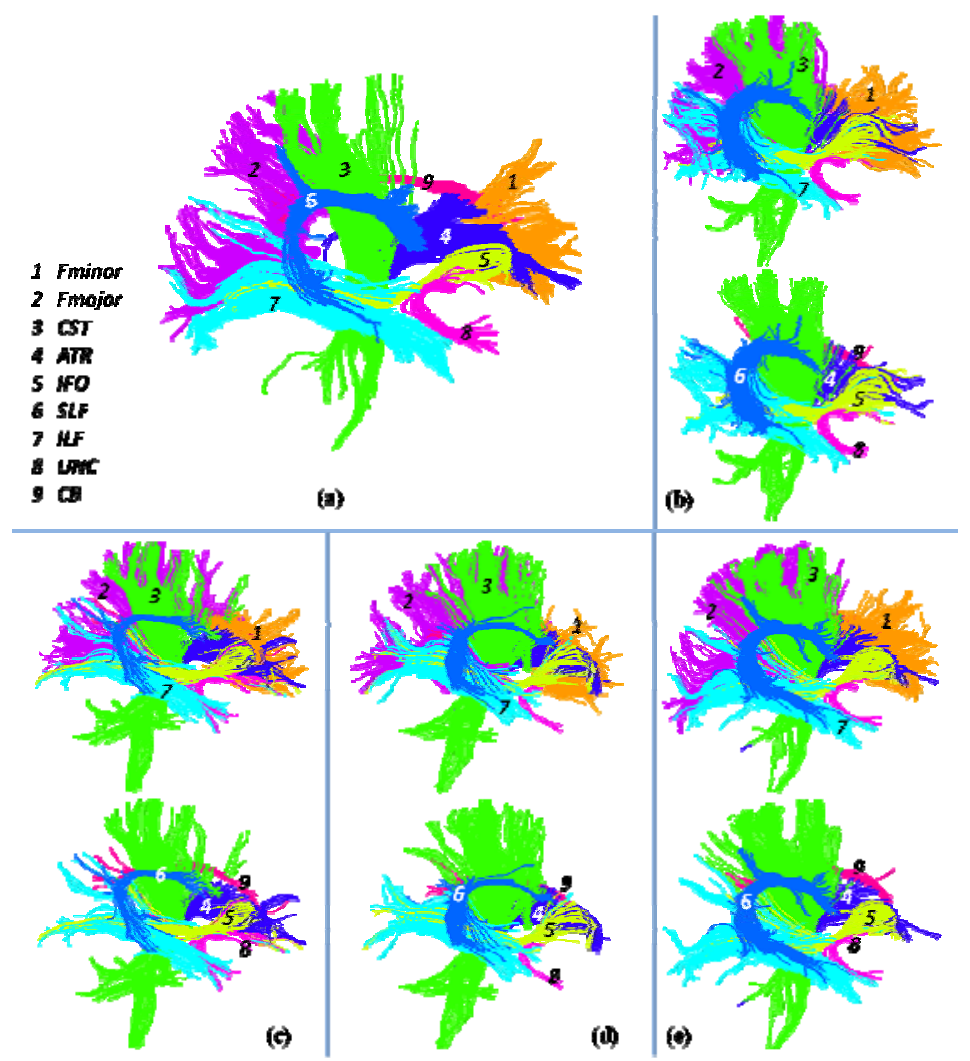

Fig. 2. Target bundles in the atlas are shown and annotated in (a). Results of 4 randomly selected subjects are shown in (b)-(e). In each panel, bundles in the right hemisphere are removed for clarity. Callosal fibers are shown only in the top images of (b)-(e), and are removed from the bottom images for better visualization.

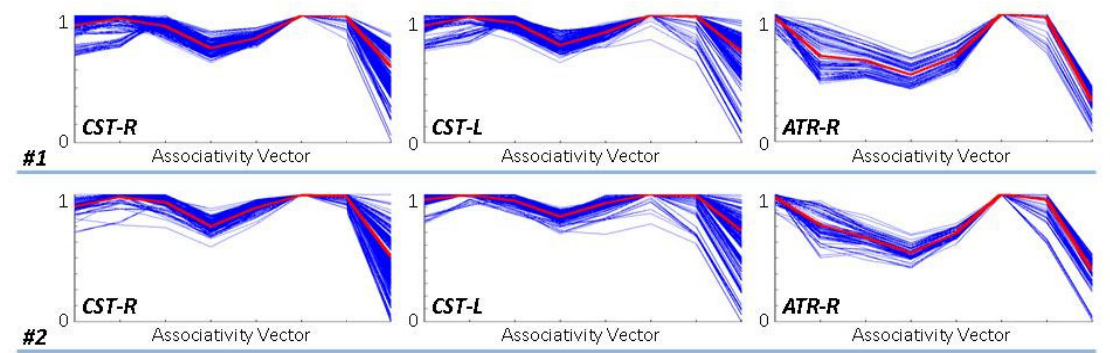

Fig. 3. The associativity vectors for CST-R, CST-L, and ATR-R from two different subjects. The red curves are the mean associativity vectors. 
In Fig. 3, we plot associativity vectors of fibers in three bundles (CST-R, CST-L, ATR-R) from two different subjects, respectively. Since the entries of the associativity vectors corresponding to the opposite hemisphere are mostly zeros, we remove them from the associativity vector before plotting. The red curves are the mean associativity vectors. For both subjects, patterns between corresponding bundles are similar to each other, indicating that clustering results are consistent across subjects. We can also observe reflectional symmetry between CST-R and CST-L. Moreover, the associativity vectors for ATR-R, which is neighboring to CST-R, show a clearly different pattern with that of CST-R, suggesting that they can be sufficiently differentiated by the bundle models during fiber clustering.

For corresponding bundles of two individual subjects, we measured their similarity using the mean of the Pearson correlation coefficients of associativity vectors between all fiber pairs. The average correlations of 16 bundles across 15 subjects, as well as standard deviations, are listed in Table 2. In most bundles, the very high correlation scores underline that clustering results are consistent across subjects, which is important to fiber clustering.

Table 2. The mean correlation of associativity vectors, as well as the standard deviation, across all 15 subjects

\begin{tabular}{ll|ll|ll}
\hline Bundle & Correlation & Bundle & Correlation & Bundle & Correlation \\
\hline Fminor & $0.891 \pm 0.016$ & IFO-R & $0.924 \pm 0.008$ & UNC-R & $0.969 \pm 0.009$ \\
Fmajor & $0.663 \pm 0.048$ & IFO-L & $0.935 \pm 0.032$ & UNC-L & $0.975 \pm 0.013$ \\
CST-R & $0.867 \pm 0.021$ & SLF-R & $0.976 \pm 0.009$ & CB-R & $0.946 \pm 0.020$ \\
CST-L & $0.906 \pm 0.020$ & SLF-L & $0.953 \pm 0.010$ & CB-L & $0.924 \pm 0.034$ \\
ATR-R & $0.898 \pm 0.022$ & IFL-R & $0.943 \pm 0.015$ & & \\
ATR-L & $0.926 \pm 0.019$ & IFL-L & $0.939 \pm 0.030$ & & \\
\hline
\end{tabular}

\section{Discussion}

In this paper, we have applied a fuzzy formulation of the associativity vector for fiber representation and modeling. Based on the associativity vectors, we model fiber bundles using multivariate Gaussian mixtures. An EM clustering scheme is employed to group the fibers into 16 major bundles. Compared with geometric methods, the proposed method relies on fiber connectivity patterns only but not geometric similarity, as complex fiber structures (fanning, branching, etc.) are challenging for modeling using geometric information. Moreover, the 16 target bundles are selected due to their easy reproducibility, though no limitation of bundles is assumed. Experimental results show that clustering results are consistent with the atlas and across subjects, which is an important property in applying this method to analysis of population data. More extensive assessment of the algorithm, however, will need to be performed. For this purpose, we will use histological data obtained from excised brains of canine subjects, which will soon be available to us from one of our projects. 


\section{References}

1. Mori, S., Zijl, P.C.M.v.: Fiber Tracking: Principles and Strategies - A Technical Review. NMR in Biomedicine 15, 468-480 (2002)

2. Ding, Z., Gore, J.C., Anderson, A.W.: Classification and Quantification of Neuronal Fiber Pathways Using Diffusion Tensor MRI. Magnetic Resonance in Medicine 49, 716-721 (2003)

3. Maddah, M., Grimson, W.E.L., Warfield, S.K., Wells, W.M.: A Unified Framework for Clustering and Quantitative Analysis of White Matter Fiber Tracts. Medical Image Analysis 12, 191-202 (2008)

4. Maddah, M., Wells III, W.M., Warfield, S.K., Westin, C.-F., Grimson, W.E.L.: Probabilistic Clustering and Quantitative Analysis of White Matter Fiber Tracts. In: Karssemeijer, N., Lelieveldt, B. (eds.) IPMI 2007. LNCS, vol. 4584, pp. 372-383. Springer, Heidelberg (2007)

5. Klein, J., Bittihn, P., Ledochowitsch, P., Hahn, H.K., Konrad, O., Rexilius, J., Peitgen, H.O.: Grid-based Spectral Fiber Clustering. In: SPIE Medical Imaging (2007)

6. Maddah, M., Crimson, W.E.L., Warfield, S.K.: Statistical modeling and EM clustering of white matter fiber tracts. In: ISBI (2006)

7. Maddah, M., Mewes, A.U.J., Haker, S., Grimson, W.E.L., Warfield, S.K.: Automated AtlasBased Clustering of White Matter Fiber Tracts from DTMRI. In: Duncan, J.S., Gerig, G. (eds.) MICCAI 2005. LNCS, vol. 3749, pp. 188-195. Springer, Heidelberg (2005)

8. Brun, A., Knutsson, H., Park, H.-J., Shenton, M.E., Westin, C.-F.: Clustering Fiber Traces Using Normalized Cuts. In: Barillot, C., Haynor, D.R., Hellier, P. (eds.) MICCAI 2004. LNCS, vol. 3216, pp. 368-375. Springer, Heidelberg (2004)

9. Klein, J., Stuke, H., Stieltjes, B., Konrad, O., Hahn, H.K., Peitgen, H.-O.: Efficient fiber clustering using parameterized polynomials. In: SPIE Medical Imaging (2008)

10. Corouge, I., Gouttard, S., Gerig, G.: Towards a Shape Model of White Matter Fiber Bundles Using Diffusion Tensor MRI. In: ISBI (2004)

11. Gerig, G., Gouttard, S., Corouge, I.: Analysis of Brain White Matter via Fiber Tract Modeling. In: IEEE EMBS (2004)

12. O’Donnell, L.J., Westin, C.F.: Automatic Tractography Segmentation Using a HighDimensional White Matter Atlas. IEEE Trans. Medical Imaging 26, 1562-1575 (2007)

13. Zhang, S., Correia, S., Laidlaw, D.H.: Identifying White-Matter Fiber Bundles in DTI Data Using an Automated Proximity-Based Fiber-Clustering Method. IEEE Trans. Visualization and Computer Graphics 14, 1044-1053 (2008)

14. Maddah, M., Zollei, L., Grimson, W.E.L., Wells, W.M.: Modeling of anatomical information in clustering of white matter fiber trajectories using Dirichlet distribution. In: MMBIA (2008)

15. Xia, Y., Turken, A.U., Whitfield-Gabrieli, S.L., Gabrieli, J.D.: Knowledge-Based Classification of Neuronal Fibers in Entire Brain. In: Duncan, J.S., Gerig, G. (eds.) MICCAI 2005. LNCS, vol. 3749, pp. 205-212. Springer, Heidelberg (2005)

16. Li, H., Xue, Z., Guo, L., Liu, T., Hunter, J., Wong, S.T.C.: A Hybrid Approach to Automatic Clustering of White Matter Fibers. NeuroImage 49, 1249-1258 (2010)

17. Wakana, S., Jiang, H., Nagae-Poetscher, L.M., van Zijl, P.C.M., Mori, S.: Fiber Tractbased Atlas of Human White Matter Anatomy. Radiology 230, 77-87 (2004)

18. Sethian, J.A.: A Fast Marching Level Set Method for Monotonically Advancing Fronts. PNAS 93, 1591-1595 (1996)

19. Wakana, S., Caprihan, A., Panzenboeck, M.M., Fallon, J.H., Perry, M., Gollub, R.L., Hua, K., Zhang, J., Jiang, H., Dubey, P., Blitz, A., van Zijl, P., Mori, S.: Reproducibility of Quantitative Tractography Methods Applied to Cerebral White Matter. NeuroImage 36, 630-644 (2007) 